\title{
Discussion on Database Design of Scientific Research Management System for Colleges and Universities
}

\author{
Zhijun CHEN \\ Scientific Research Division of Neijiang Normal University, Neijiang, China
}

\begin{abstract}
As scientific research of colleges and universities plays an increasingly significant role in scientific and technological progress of the state, it is extremely urgent to establish a scientific research management system for colleges and universities. This paper describes the design method and key technology to realize database of scientific research management system for colleges and universities; the system can adapt to management requirements regarding maintenance, check and statistics, report and analysis of scientific research information as well as application, approval, conclusion etc. of university-level topics, methodologies and mechanisms such as referential integrity, storage process, trigger and affair etc.
\end{abstract}

KEYWORD: Database; Scientific Research Management; Instances

\section{INTRODUCTION}

With continuous development of the society, the level of scientific research has become an important measure of colleges and universities' strength. As an important scientific research institution, how does the colleges and universities handle the numerous management work including saving, handling, statistics and processing of scientific research information? so that the daily scientific research management can be more standardized, scientific and efficient, and the establishment of a good scientific research management system for colleges and universities is a way that must be passed by each of the universities. The relation between system function analysis and data structure as well as its application are first reflected in design of the database, and database design for scientific research management system of colleges and universities is a core work in design of the management system, so all the management work shall be based on the database.

\section{DEMAND ANALYSIS}

Design of scientific research in colleges and universities is very complicated and involves scientific research management of the Scientific Research Division and various departments in the university and personal check and statistics of scientific research information [1]. The system designed shall both be beneficial to supervision and management of the Scientific Research Division and in favor of division and coordination of departments and teachers. The system mainly includes the following functions: scientific research management of the Scientific Research Division, scientific research management of colleges (or departments), check of personal scientific research information and overall information management of university-level scientific research projects. It consists of maintenance of scientific research information, check and statistics of scientific research information, report of scientific research information, analysis of scientific research information, application, approval and conclusion of university-level topics and other processing, among them, scientific research information is further divided into academic paper, scientific research project, scientific research achievements and reward for scientific research etc.

\section{DATABASE DESIGN}

\subsection{Conceptual Structure Design}

Actually, conceptual structure design is to abstract the user demand derived from system demand analysis into an information structure. Result of the conceptual design is conceptual model of the database which is illustrated by Fig. E-R. Firstly, we establish a relation model for the whole system according to the following steps: [2] 
(1) Instance Diagram of Information in Table of Participation Achievements Information: (Fig. 1)

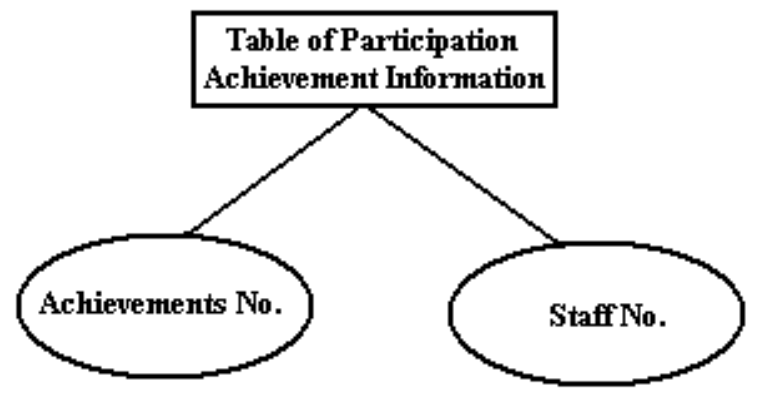

Fig. 1 Instance Diagram of Participation Achievements

(2) Instance Diagram of Teacher Information: (Fig. 2)

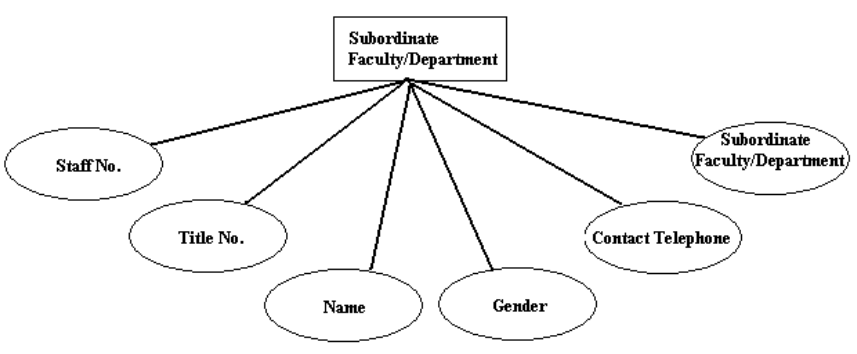

Fig. 2 Instance Diagram of Scientific Research Awards

(3) Instance Diagram for Table of Scientific Research Achievements Registration Information (Fig. 3)

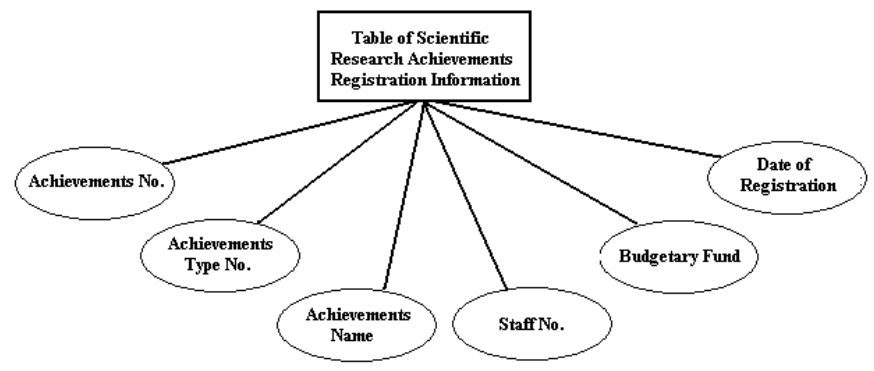

Fig. 3 Instance Diagram of Achievements Type

(4) Instance Diagram for Table of Scientific Research Achievements Conclusion: (Fig. 4)

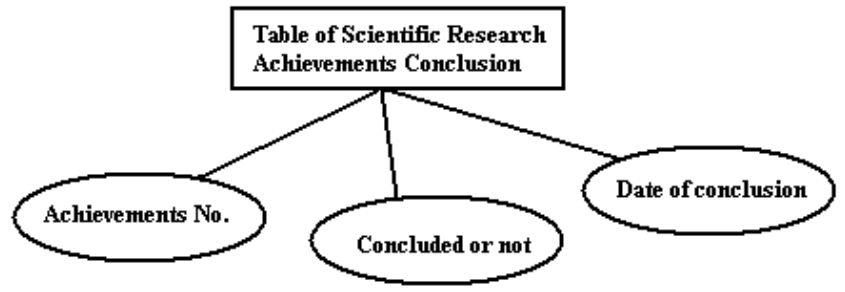

Fig. 4 Instance Diagram of Department Information

(5) Instance Diagram for Table of Scientific Research Achievements Information: (Fig. 5)

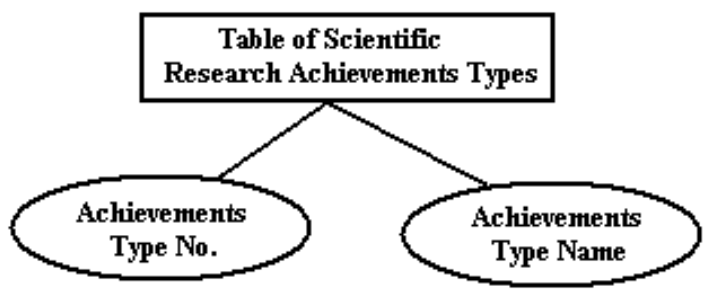

Fig. 5 Instance Diagram of Teacher Information

(6) Instance Diagram for Table of Scientific Research Achievements Review Information: (Fig.6)

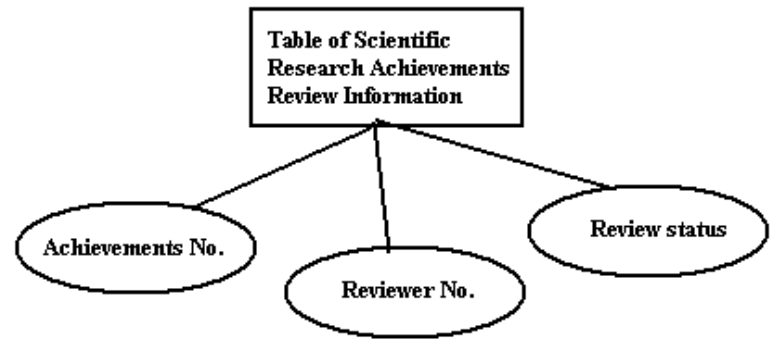

Fig. 6 Instance Diagram of Achievements Information

(7) Instance Diagram for Table of Scientific Research Reward Information: (Fig. 7)

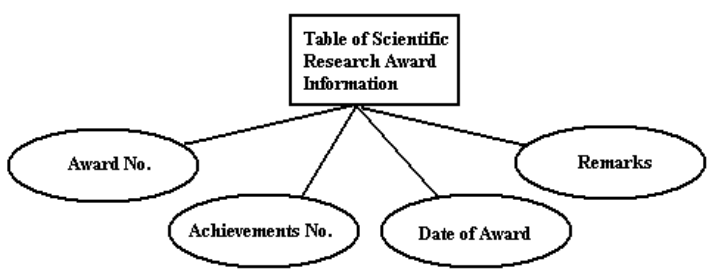

Fig. 7 Instance Diagram of Scientific Research Projects

(8) Instance Diagram for Table of Scientific Research Staff Information: (Fig. 8)

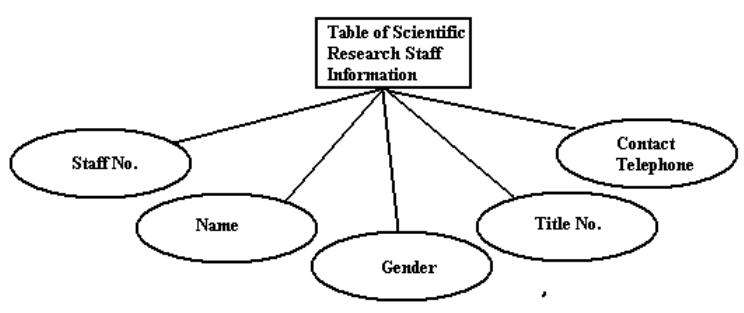

Fig. 8 Instance Diagram of Paper Information

(9) Model Diagram for Table of Staff Title Information: (Fig. 9)

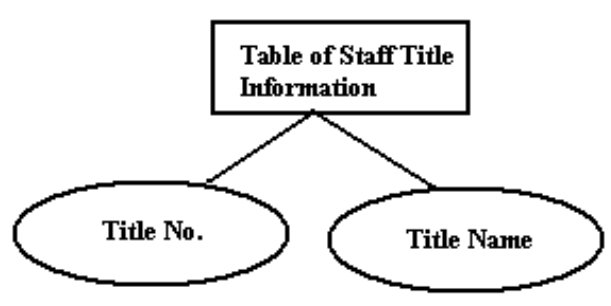

Fig. 9 Instance Diagram of Participation Achievements 
(10) Model Diagram for Table of Department Information: (Fig. 10)

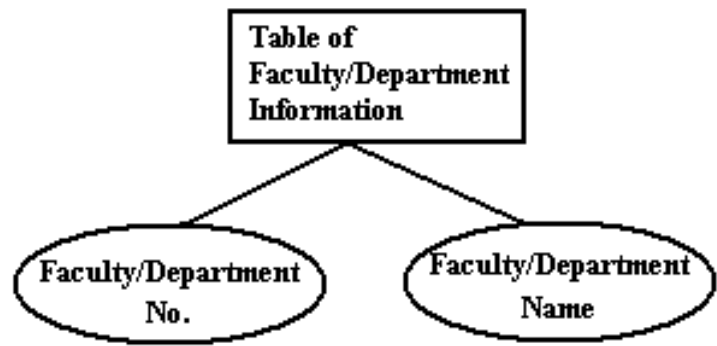

Fig. 10 Instance Diagram of Reviewed Achievements

(11) General Diagram E-R: (Fig. 11))

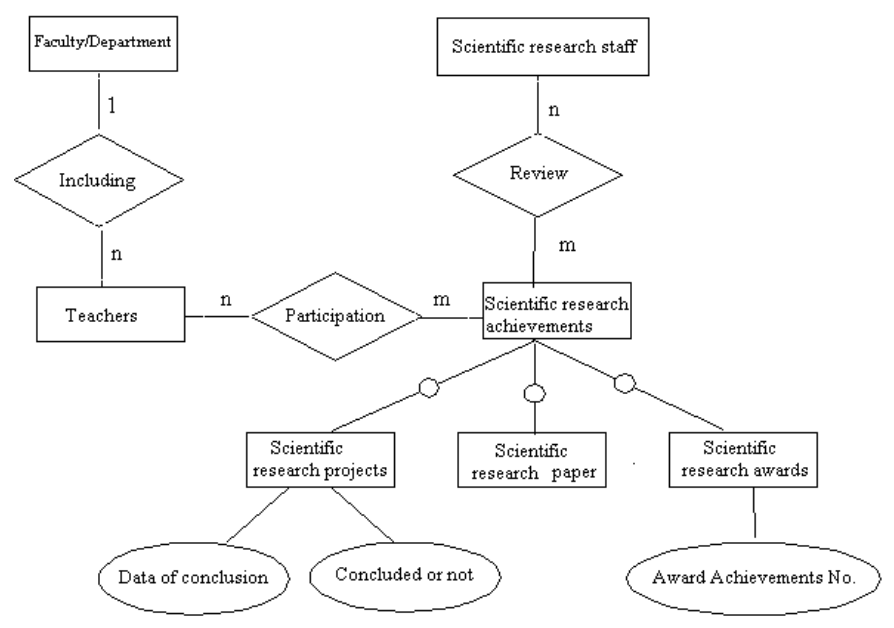

Fig. 11 General Diagram E-R

\subsection{Logical Structure Design}

Based on conceptual structure design of the system, the system is realized directly through abstract positioning on better adaption to demand of and operation by persons of all levels, and all initialized data of the system are input through interface by various persons through division of labor. According to actual situation of our university, the writer treats basic information of the scientific research staff, achievements information, awards information, department information and teacher information etc. as initialized data of the system so as to provide a basic data platform for check and statistics by teachers and departments as well as for operation, processing, management and integration etc. by the Scientific Research Division. [3]

\subsection{Storage Process Design}

The storage process is an integration of TransactSQL words and sentences, it is a good way to realize affair or business rules and it is implemented together with saving in the data server. Use of the procedure can significantly reduce network transmission and improves performance and security of application programs; in addition, it is optimized and compiled only for first implementation; therefore, use of the storage process can not only greatly reduce the difficulty in realization of application programs, but also significantly improve running speed and efficiency of the system. Design of the storage process must conform to rules and requirements of logic and business, and some regular and frequently check and insertion data operation can be finished by the storage process.

\subsection{View Mechanism Design}

View is a logic window consisting of data selected from basic table of the database, and the difference from the basic table is that view is a virtual table. Instead of data contained in the view, the database stores definition of the view only, and such data are still stored in its original basic table. Therefore, if data change in the basic table, data checked in the view also change accordingly. In this sense, a view is a window through which data in the database to which the user is interested can be checked. [4]

\subsection{Database Security Design}

As network becomes increasingly generalized, network security will become more and more important, taking database level, server level and application program level into overall consideration, different authority levels and different initializing menus shall be set in scientific research management system. In this system, the database security is mainly realized through access control mechanism of the database. It defines operating authorization, i.e. role of various administers, and then defines log-in level of the database, finally distributes the log-in levels into corresponding roles based on table of user authorization. Due to complexity of persons, the roles are divided into three levels: the Scientific Research Division level, department level and ordinary teacher level. Users belongs to a certain role, i.e. even in case of users' job transfer, the addition or deletion of the user won't affect operation of other users, and the only change lies in number of users in the role. A special table of system utilization authorization is established to record users and their authorization, and integrate department information at the same time, distribute corresponding authorization and account number of different departments, and the Scientific Research Division enjoys the highest management level. [5]

This system database is mainly applicable to database system of $\mathrm{C} / \mathrm{s}$ and $\mathrm{B} / \mathrm{s}$ mixed mode. Design shall be conducted in overall consideration to create a strict and scientific database structure with referential integrity, with storage process and trigger applied in database design, the backstage function and efficiency are greatly improved, and the classified and categorized set and management of the 
database security also improves its performance of safe access; with little data redundancy, it has even higher level of data consistency, operability and security, and the combination with front-stage development tools and platforms, it can realize powerful functional design and system.

\section{REFERENCES}

[1] REN Rong, BAO Wen-xin. The Research and Realization of the Science Feed Management and Quality Attestation
System in Livestock Norm Production. Journal of Chongqing Normal University, 2009, 26(4).

[2] Chen-Feng Wu. Design of Portable Personal Information Management System with XML Technique. Information Technology Journal, 2008, 7(4).

[3] Chengping Ruan, Shiming Dai, Chengshen Ruan. Study on Agricultural Resource Management Information System in Huairou District. Scientific Journal of Information Engineering, 2013, 2(2).

[4] Ugur Demiray. From Editor vol 11, No.3.The Turkish Online Journal of Distance Education, 2010, 11(3).

[5] Chen, Wenjin, Schmidt, Cristina, Parashar, Manish. Decentralized Data Sharing of Tissue Microarrays for Investigative Research in Oncology. 2008. 